\title{
CONSTANTS OF DERIVATIONS ON FREE ASSOCIATIVE ALGEBRAS
}

\author{
VITOR DE OLIVEIRA FERREIRA* \\ Departamento de Matemática, Instituto de Matemática e Estatística, Universidade de São Paulo, Caixa \\ Postal 66281, São Paulo - SP, 05315-970, Brazil \\ e-mail:vofer@ime.usp.br
}

(Received 13 June, 2000; accepted 17 October 2000)

\begin{abstract}
It is proved that the subalgebra of constants of a derivation on a free associative algebra in prime characteristic is free provided that some constraining conditions are satisfied. As a particular case, it follows that the constants of the partial derivatives on a free algebra form a free subalgebra. The main result is also applied in order to provide a simplified proof of a previous result by the author on extensions of tensor rings.
\end{abstract}

2000 Mathematics Subject Classification. 16S10, 16W25.

0. Introduction. It is a known fact that the subalgebra of constants of any Lie algebra of homogeneous derivations on a free associative algebra is free (see $[\mathbf{5 , 6}]$ ). G. Bergman gave the following example of a derivation on a free algebra whose subalgebra of constants is not free. Consider the free associative algebra of rank 3 over a field $k, R=k\langle x, y, z\rangle$, and let $d$ be the $k$-derivation on $R$ given by

$$
d(x)=x y x+x, \quad d(y)=-y x y-y, \quad d(z)=-x .
$$

In $[7,6.1 .12]$, V. Kharchenko indicates how to show that $\operatorname{ker}(d)$ is not a free subalgebra. It is also noted that if the characteristic of $k$ is positive, then the restricted Lie algebra generated by $d$ is infinite dimensional. In view of these facts, Kharchenko formulates the following question: "Will an algebra of constants of a finitedimensional restricted Lie algebra of derivations of a free algebra over a field of positive characteristic be free?" ([7, 6.1.14]).

In this paper we deal with derivations which are not homogeneous, but satisfy the condition that the subalgebras of lower rank are invariant under them. We give sufficient conditions on a nilpotent derivation on a free algebra (over a field of positive characteristic) for the subalgebra of constants to be free (Theorem 5).

By a result of Lam and Leroy (Theorem 1), certain Ore extension quotients defined by derivations satisfying the hypotheses of Theorem 5 give rise to complete $p^{n} \times p^{n}$ matrix rings over the constants. In the proof of Theorem 5 we use the given derivation in order to construct an Ore extension and then use Theorem 1 in order to characterize the ring of constants as the base ring of a matrix ring. On the other hand, using a result on extensions of matrix rings (Lemma 4), we find an

*The author was supported by FAPESP - São Paulo/Brazil, Grant 98/14403-8. 
isomorphism which preserves matrix units between the matrix ring over the constants and a matrix ring over a free algebra.

A direct application of the main theorem is the fact that the constants of the partial derivatives on a free algebra form a free subalgebra (Corollary 6).

In [3, Theorem 3.2] the author showed that, given a field $k$ of positive characteristic $p$ and a simple purely inseparable extension $F$ of $k$ of degree $p$, the extension of the free $F_{k}$-ring by $F$ over $k$ was not a $p \times p$ matrix ring over a free ideal ring, but that there existed a finite localization of it with this property. In the original article, this result was proved using methods of recognition of matrix units and actual computation of the centralizer of the matrix units. Here we use Corollary 7 of Theorem 5 to provide a simpler proof of this result (Theorem 8).

Throughout this paper all rings are associative, but not necessarily commutative, and have an identity element which is inherited by subrings and preserved by homomorphisms. We use the convention that fields are not necessarily commutative. The set of all non-negative integers will be denoted by $\mathbf{N}$.

1. Preliminaries. Given a ring $R$, by a derivation on $R$ one understands an additive map $\delta: R \longrightarrow R$ which satisfies $\delta(a b)=\delta(a) b+a \delta(b)$ for $a, b \in R$. If $R$ is an algebra over a field $k$, then by a $k$-derivation on $R$ it will be understood a derivation which is $k$-linear. If $\delta$ is a derivation on $R$, then $\operatorname{ker}(\delta)=\{a \in R: \delta(a)=0\}$ is a subring of $R$, which is called the subring of constants of $R$ under $\delta$.

We shall be looking at derivations on free associative algebras. It is known that in order to define a $k$-derivation $\delta$ on a free associative algebra $k\langle X\rangle$ it is enough to describe the image of $\delta$ on the free generators $x \in X$.

Given a ring $R$ and a derivation $\delta$ on $R$, the Ore extension $R[t, \delta]$ is defined to be the ring generated by $t$ over $R$ with defining relations

$$
t a=a t+\delta(a)
$$

for all $a \in R$. So the elements of $R[t, \delta]$ have a normal form given by the polynomials $\sum_{i=0}^{n} a_{i} t^{i}$, where $n \in \mathbf{N}$ and $a_{i} \in R$, and multiplication is induced by (1).

Our purpose here is to investigate subalgebras of constants of derivations on free associative algebras. We shall make use of the following result by Lam and Leroy which captures subrings of constants of derivations satisfying some conditions.

Theorem 1 ([8, Theorem 3.1]). Let $R$ be a ring of prime characteristic $p$, and let $\delta$ be a derivation on $R$ such that $\delta^{p^{n}}=0$ and $1 \in \delta^{p^{n-1}}(R)$, where $n$ is a fixed positive integer. Consider the Ore extension $R[t, \delta]$, and let $T=R[t, \delta] /\left\langle t^{p^{n}}\right\rangle$. Then $T \cong \mathbf{M}_{p^{n}}\left(R_{\delta}\right)$, where $R_{\delta}$ is the subring of constants of $R$ under the derivation $\delta$.

In [8, Example 3.4] the authors use Theorem 1 to show that, for a field $k$ of positive characteristic $p$, there is an isomorphism between the "truncated" first Weyl algebra $k[x]\left[t, \frac{d}{d x}\right] /\left\langle t^{p}\right\rangle$ and the full $p \times p$ matrix ring over a polynomial ring $k[x]$ in one indeterminate. In fact, Theorem 1 can be used to prove that $k[x][t, \delta] /\left\langle t^{p^{n}}\right\rangle \cong$ $\mathbf{M}_{p^{n}}(k[x])$, for any derivation $\delta$ such that $\delta^{p^{n}}=0$ and $1 \in \delta^{p^{n-1}}(k[x])$. Indeed, if $\delta$ is a nonzero derivation on $k[x]$, then, for every $f \in k[x]$, we have $\delta(f)=\frac{d f}{d x} \cdot \delta(x)$. So $\delta(f)=0$ if and only if $f \in k\left[x^{p}\right] \cong k[x]$. 
REMARK 2. The proof of Theorem 1 is based on the proof of [1, Theorem 3.1] which, in turn, uses the fact that under these hypotheses there exist $a, b \in T$ such that $a f^{p^{n}-1}+f b=1$, where $f$ is the image of $t$ in $T$. It is this equation and the fact that $f^{p^{n}}=0$ that are used with [1, Theorem 1.1] to characterize $T$ as a $p^{n} \times p^{n}$ matrix ring. Following the proof of this result, [1, Note 1.2] shows that the matrix corresponding to $f$, in this context, is given by $e_{21}+e_{32}+\ldots+e_{p^{n}, p^{n}-1}$, where $e_{i j}$ are the matrix units which being present in $T$ make it isomorphic to a full $p^{n} \times p^{n}$ matrix ring. Hence $f$ corresponds to a $p^{n} \times p^{n}$ matrix $[f]$ with entries in the ground field $k$ and which is such that $[f]^{p^{n}}=0$, but $[f]^{p^{n}-1} \neq 0$. This fact will be widely used in what follows.

We shall make use of the following generalization of a result by Frobenius. (The original formulation can be found in [3, Lemma 3.4].)

Lemma 3. Let $k$ be a field, let $R$ be a $k$-algebra, let $S$ be a subalgebra of $R$, and let $n$ be a positive integer. Let $A \in \mathbf{M}_{n}(k)$ be such that $A^{n}=0$, but $A^{n-1} \neq 0$. Suppose that $B \in \mathbf{M}_{n}(R)$ is such that $A B-B A \in \mathbf{M}_{n}(S)$. Then there exist $r_{1}, \ldots, r_{n} \in R$ such that $B-\sum_{i=1}^{n} r_{i} A^{i-1} \in \mathbf{M}_{n}(S)$.

Proof. Since $A^{n}=0$, but $A^{n-1} \neq 0$, there exists a vector $v \in k^{n}$ such that $\left\{v, A v, \ldots, A^{n-1} v\right\}$ is a basis for $k^{n}$ over $k$ and therefore a basis for $R^{n}$ over $R$. So there exist $r_{1}, \ldots, r_{n} \in R$ such that $B v=\sum_{i=1}^{n} r_{i} A^{i-1} v$, or, in a form that will be more useful later,

$$
\left(B-\sum_{i=1}^{n} r_{i} A^{i-1}\right) v=0 .
$$

Let $C=A B-B A$ and $\left\{C_{j}: j \geq 1\right\}$ be the family of matrices over $S$ defined recursively by $C_{1}=C$ and $C_{j}=A^{j-1} C+C_{j-1} A$ for $j>1$. It can be easily proved by induction on $j$ that $B A^{j}=A^{j} B-C_{j}$. For each $j=1, \ldots, n-1$, we have

$$
\begin{aligned}
\left(B-\sum_{i=1}^{n} r_{i} A^{i-1}\right) A^{j} v & =\left(B A^{j}-A^{j} \sum_{i=1}^{n} r_{i} A^{i-1}\right) v \\
& =A^{j}\left(B-\sum_{i=1}^{n} r_{i} A^{i-1}\right) v-C_{j} v
\end{aligned}
$$

The first equality follows from the fact that, since $A$ has entries in $k$, the $r_{i}$ commute with $A$. By (2), it follows that

$$
\left(B-\sum_{i=1}^{n} r_{i} A^{i-1}\right) A^{j} v=-C_{j} v
$$

for $j=1, \ldots, n-1$. Let $\left\{e_{1}, \ldots, e_{n}\right\}$ be the canonical basis of $k^{n}$ over $k$ and denote by $P$ the invertible matrix over $k$ defined by $P e_{j}=A^{j-1} v$, for $j=1, \ldots, n$. So, setting $\Delta_{j}=-C_{j} P$, we can rewrite (3) as

$$
\left(B-\sum_{i=1}^{n} r_{i} A^{i-1}\right) P e_{j+1}=\Delta_{j} e_{1} .
$$


Let $\Delta_{j}^{0}$ be the first column of $\Delta_{j}$ and denote by $\Delta$ the matrix whose first column is 0 and the other columns are the $\Delta_{j}^{0}$, that is,

$$
\Delta=\left(\begin{array}{lllll}
0 & \Delta_{1}^{0} & \Delta_{2}^{0} & \cdots & \Delta_{n-1}^{0}
\end{array}\right) .
$$

Since, by hypothesis, $C \in \mathbf{M}_{n}(S)$, and both $A$ and $P$ have entries in $k$, it follows that $\Delta \in \mathbf{M}_{n}(S)$. Equations (2) and (4) amount to $\left(B-\sum_{i=1}^{n} r_{i} A^{i-1}\right) P=\Delta$. Thus

$$
B-\sum_{i=1}^{n} r_{i} A^{i-1}=\Delta P^{-1} \in \mathbf{M}_{n}(S) .
$$

And this proves the lemma.

2. Main result. Given a field $k$ and $k$-algebras $R$ and $S$, we denote by $R *_{k} S$ the coproduct of $R$ and $S$ in the category of $k$-algebras. Briefly, $R *_{k} S$ is the $k$-algebra whose generators are the generators of $R$ and of $S$, and whose defining relations are those holding in $R$, in $S$, and those that identify element-wise the copy of $k$ in $R$ with the copy of $k$ in $S$. For example, $k[x] *_{k} k[y] \cong k\langle x, y\rangle$.

We are ready to state and prove a lemma which will lead us to the main result of the paper.

Lemma 4. Let $k$ be a field, let $T$ be a $k$-algebra, and let $n$ be a positive integer. Let $\xi \in \mathbf{M}_{n}(k)$ be such that $\xi^{n}=0$, but $\xi^{n-1} \neq 0$, and let $r \in \mathbf{M}_{n}(T)$ be an arbitrary matrix. Then, there is a matrix-units-preserving isomorphism

$$
\frac{\mathbf{M}_{n}(T) *_{k} k[x]}{\langle\xi x-x \xi-r\rangle} \cong \mathbf{M}_{n}\left(\bar{T} *_{k} k\left\langle z_{1}, \ldots, z_{n}\right\rangle\right),
$$

where $\bar{T}=T / I$ and $I$ is the kernel of the canonical homomorphism

$$
T \longrightarrow \mathbf{M}_{n}(T) \longrightarrow \mathbf{M}_{n}(T) *_{k} k[x] \longrightarrow \frac{\mathbf{M}_{n}(T) *_{k} k[x]}{\langle\xi x-x \xi-r\rangle},
$$

which depends on the choice of $r$.

Proof. Let $S=\left(\mathbf{M}_{n}(T) *_{k} k[x]\right) /\langle\xi x-x \xi-r\rangle$. The image of the matrix units of $\mathbf{M}_{n}(T)$ in $S$ form a complete set of $n \times n$ matrix units for $S$; so $S$ is isomorphic to a full $n \times n$ matrix ring over some $k$-algebra $A$ containing $\bar{T}$. Given an element $s \in \mathbf{M}_{n}(T) *_{k} k[x]$, let $\bar{s}$ denote its image in $S \cong \mathbf{M}_{n}(A)$. With this notation, given an element $a \in \mathbf{M}_{n}(T), \bar{a}$ coincides, via the identification of $S$ with $\mathbf{M}_{n}(A)$, with the element of $\mathbf{M}_{n}(\bar{T})$ obtained from $a$ by projecting its entries onto $\bar{T}$. Thus, in $\mathbf{M}_{n}(A)$, the following identity holds:

$$
\xi \bar{x}-\bar{x} \xi=\bar{r} \in \mathbf{M}_{n}(\bar{T}) .
$$

By Lemma 3, there exist $a_{1}, \ldots, a_{n} \in A$ and $D \in \mathbf{M}_{n}(\bar{T})$ such that

$$
\bar{x}=\sum_{i=1}^{n} a_{i} \xi^{i-1}+D .
$$


Since $a_{i} \in A$, it commutes with $\xi$ and so $\xi D-D \xi=\bar{r}$. The maps $\bar{T} \hookrightarrow A$ and $k\left\langle z_{1}, \ldots, z_{n}\right\rangle \longrightarrow A$, sending $z_{i}$ to $a_{i}$, induce an $\mathbf{M}_{n}(T)_{k}$-ring homomorphism

$$
\varphi: \mathbf{M}_{n}\left(\bar{T} *_{k} k\left\langle z_{1}, \ldots, z_{n}\right\rangle\right) \longrightarrow \mathbf{M}_{n}(A) \cong S
$$

On the other hand, there is a unique $\mathbf{M}_{n}(T)_{k}$-ring homomorphism $\hat{\psi}$ from the free $\mathbf{M}_{n}(T)_{k}$-ring $\mathbf{M}_{n}(T) *_{k} k[x]$ into $\mathbf{M}_{n}\left(\bar{T} *_{k} k\left\langle z_{1}, \ldots, z_{n}\right\rangle\right)$ sending $x$ to $\sum_{i=1}^{n} z_{i} \xi^{i-1}+D$. This induces a homomorphism

$$
\psi: S \longrightarrow \mathbf{M}_{n}\left(\bar{T} *_{k} k\left\langle z_{1}, \ldots, z_{n}\right\rangle\right),
$$

for

$$
\begin{aligned}
\hat{\psi}(\xi x-x \xi-r) & =\xi \hat{\psi}(x)-\hat{\psi}(x) \xi-\bar{r} \\
& =\xi\left(\sum_{i} z_{i} \xi^{i-1}+D\right)-\left(\sum_{i} z_{i} \xi^{i-1}+D\right) \xi-\bar{r} \\
& =\xi D-D \xi-\bar{r} \\
& =0 .
\end{aligned}
$$

We now show that $\psi$ is the inverse of $\varphi$. Indeed, on the one hand, $\sum_{i} z_{i} \xi^{i-1}+D=\psi(x)=\psi\left(\sum_{i} a_{i} \xi^{i-1}+D\right)=\sum_{i} \psi\left(a_{i}\right) \xi^{i-1}+D . \quad$ So $\quad \sum_{i} z_{i} \xi^{i-1}=$ $\sum_{i} \psi\left(a_{i}\right) \xi^{i-1}$. Multiplying this last equation by $\xi^{n-1}$ yields $z_{1} \xi^{n-1}=\psi\left(a_{1}\right) \xi^{n-1}$. Since $\xi^{n-1} \neq 0$ and its entries lie in $k$, it follows that $z_{1}=\psi\left(a_{1}\right)$. Analogously, one shows that $z_{i}=\psi\left(a_{i}\right)$ for $i=2, \ldots, n$. Therefore, $\varphi \psi(x)=\varphi\left(\sum_{i} z_{i} \xi^{i-1}+D\right)=$ $\sum_{i} \varphi\left(z_{i}\right) \xi^{i-1}+D=\sum_{i} a_{i} \xi^{i-1}+D=x$. On the other hand, it is clear that $\psi \varphi\left(z_{i}\right)=z_{i}$, for all $i=1, \ldots, n$. Thus $\varphi$ is an isomorphism.

If in Lemma 4 the canonical map $\mathbf{M}_{n}(T) \longrightarrow S$ is injective, then there exists an isomorphism between $S$ and $\mathbf{M}_{n}\left(T *_{k} k\left\langle z_{1}, \ldots, z_{n}\right\rangle\right)$. This will be the case of interest to us in what follows.

THEOREM 5. Let $k$ be a field of positive characteristic $p$, let $m, n$ be positive integers and let $\delta$ be a k-derivation on the free associative algebra of rank $m$, $R=k\left\langle x_{1}, \ldots, x_{m}\right\rangle$. Suppose that

(1) $\delta^{p^{n}}=0$,

(2) $\delta\left(x_{1}\right) \in k\left[x_{1}\right]$ and $\delta\left(x_{i}\right) \in k\left\langle x_{1}, \ldots, x_{i-1}\right\rangle$ for $i=2, \ldots, m$, and

(3) $1 \in \delta^{p^{n-1}}\left(k\left[x_{1}\right]\right)$.

Then the subalgebra of constants $\operatorname{ker}(\delta)$ of $R$ under the derivation $\delta$ is free of rank $(m-1) p^{n}+1$.

Proof. We shall proceed by induction on $m$. We know the result is true for $m=1$, for any derivation on $k\left[x_{1}\right]$ is a multiple of $\frac{d}{d x_{1}}$. Suppose the result holds for $m>1$ and let us show that it holds for $m+1$. Let $K_{m}=k\left\langle x_{1}, \ldots, x_{m}\right\rangle$ and $\delta_{m}=\left.\delta\right|_{K_{m}}$. By hypothesis (2), $\delta_{m}$ is a derivation on $K_{m}$. Consider the ring $R_{m}=\left(K_{m}\left[t, \delta_{m}\right]\right) /\left\langle t^{p^{m^{m}}}\right\rangle$. By Theorem $1, R_{m} \cong \mathbf{M}_{p^{n}}\left(\operatorname{ker}\left(\delta_{m}\right)\right)$. Let $R_{m+1}=\left(K_{m+1}[t, \delta]\right) /\left\langle t^{p^{n}}\right\rangle$, where $K_{m+1}=$ $k\left\langle x_{1}, \ldots, x_{m+1}\right\rangle$. It is immediate to see that

$$
R_{m+1} \cong \frac{R_{m} *_{k} k\left[x_{m+1}\right]}{\left\langle\xi x_{m+1}-x_{m+1} \xi-\delta\left(x_{m+1}\right)\right\rangle},
$$


where $\xi$ denotes the image of $t$ in $R_{m}$. Since $R_{m} \subseteq R_{m+1}$ and $R_{m} \cong \mathbf{M}_{p^{n}}\left(\operatorname{ker}\left(\delta_{m}\right)\right)$, it follows that $\operatorname{ker}\left(\delta_{m}\right) \subseteq R_{m+1}$. By Remark 2, the matrix corresponding to $\xi$ in $R_{m}$ is nilpotent of index $p^{n}$ over $k$. Therefore, by Lemma $4, R_{m+1} \cong \mathbf{M}_{p^{n}}\left(\operatorname{ker}\left(\delta_{m}\right) *_{k}\right.$ $\left.k\left\langle z_{1}, \ldots, z_{p^{n}}\right\rangle\right)$. On the other hand, by Theorem $1, R_{m+1} \cong \mathbf{M}_{p^{n}}(\operatorname{ker}(\delta))$ and by the induction hypothesis $\operatorname{ker}\left(\delta_{m}\right)$ is isomorphic to $k\left\langle y_{1}, \ldots, y_{(m-1) p^{n}+1}\right\rangle$. Thus,

$$
\mathbf{M}_{p^{n}}\left(k\left\langle y_{1}, \ldots, y_{(m-1) p^{n}+1}, z_{1}, \ldots, z_{p^{n}}\right\rangle\right) \cong R_{m+1} \cong \mathbf{M}_{p^{n}}(\operatorname{ker}(\delta)) .
$$

Since the above isomorphism preserves matrix units, $\operatorname{ker}(\delta)$ is isomorphic to the free associative algebra of $\operatorname{rank}(m-1) p^{n}+1+p^{n}=m p^{n}+1$ over $k$. This proves the theorem.

3. Applications. We present two immediate applications of Theorem 5. The second of these (Corollary 7) allows us to give a simplified proof of [3, Theorem 3.2].

By the partial derivative with respect to $x_{i}$ of $k\left\langle x_{1}, \ldots, x_{m}\right\rangle$, one understands the $k$-derivation $\partial_{i}$ on $k\left\langle x_{1}, \ldots, x_{m}\right\rangle$ given by $\partial_{i}\left(x_{j}\right)=\delta_{i j}$. We start by noting that the partial derivatives of a free associative algebra in prime characteristic satisfy the hypothesis of Theorem 5 (after a suitable rearrangement of the $x_{i}$ ). So we have the following result.

COROLlary 6. The subalgebra of constants of each partial derivative of the free associative algebra of rank $m$ over a field of positive characteristic $p$ is free of rank $(m-1) p+1$.

In fact, Corollary 6 is well-known (see, [5,4]). Using ideas from [4], one can prove that, in characteristic 0 , the subalgebra of constants of the partial derivative of $k\langle X\rangle$ with respect to some $x \in X$ is freely generated by the set $\left\{(\operatorname{ad} x)^{i} z\right.$ : $i \in \mathbf{N}, z \in X \backslash\{x\}\}$, where $(\operatorname{ad} x)$ is the inner derivation of $k\langle X\rangle$ determined by $x$, that is, $(\operatorname{ad} x) f=x f-f x$ for all $f \in k\langle X\rangle$. It is not difficult to see that in characteristic $p>0$ a free generating set is given by $\left\{x^{p},(\operatorname{ad} x)^{i} z: i=0, \ldots, p-1, z \in X \backslash\{x\}\right\}$.

Next, note that the $k$-derivation $\delta$ on $k\left\langle x_{1}, \ldots, x_{p-1}\right\rangle$ given by $\delta\left(x_{i}\right)=x_{i-1}$, for $i=2, \ldots, p-1$, and $\delta\left(x_{1}\right)=1$ also satisfies the hypothesis of Theorem 5 . So the following is also true.

COROLlary 7. Let $k$ be a field of positive characteristic $p$ and let $\delta$ be the derivation on $k\left\langle x_{1}, \ldots, x_{p-1}\right\rangle$ defined by $\delta\left(x_{i}\right)=x_{i-1}$ for $i=2, \ldots, p-1$, and $\delta\left(x_{1}\right)=1$. Then the subalgebra of constants of $\delta$ is free of rank $p^{2}-2 p+1$.

Incidentally, Corollary 7 is exactly what is needed to prove our next result. First, recall that, given a field $F$ (commutative or not) and a subfield $k$, the free $F_{k}$-ring on a set $X$ is defined to be the ring $F_{k}\langle X\rangle$ generated by $X$ over $F$ with defining relations $x \lambda=\lambda x$, for $x \in X$ and $\lambda \in k$. It follows that $F_{k}\langle X\rangle \cong F *_{k} k\langle X\rangle$. Because $F_{k}\langle X\rangle$ is a fir ([2, Theorem 2.6.2]), it has a universal field of fractions ([2, Corollary 7.5.11]).

Theorem 8 ([3, Theorem 3.2]). Let $k$ be a field of positive characteristic $p$ and let $F=k(\alpha)$, where $\alpha^{p} \in k$, but $\alpha \notin k$. Let $R=F_{k}\langle x\rangle$ and denote by $U$ its universal field of fractions. Then there exists a subring $S$ of $U$, obtained from $R$ by adjoining the inverse of a single element, such that $S \otimes_{k} F$ is isomorphic to $\mathbf{M}_{p}(A)$, where 


$$
A=F\left\langle z_{1}, \ldots, z_{p^{2}-p}\right\rangle *_{F} F\left[w, w^{-1}\right] .
$$

Proof. We use the notation $S_{F}=S \otimes_{k} F$. As in [3], we adjoin to $R$ the inverse of $d_{\alpha}^{p-1}(x)$, where $d_{\alpha}$ denotes the inner derivation of $R$ determined by $\alpha$, that is, $d_{\alpha}(r)=\alpha r-r \alpha$ for all $r \in R$. We can, then, write

$$
S_{F} \cong F\left\langle\xi, x, y \mid \xi^{p}=0, d^{p-1}(x) y=y d^{p-1}(x)=1\right\rangle,
$$

where $d$ is the inner derivation of $F\langle\xi, x, y\rangle$ determined by $\xi$. It is easy to see that the map sending $x$ to $u_{p-1} v$ induces an isomorphism from $S_{F}$ onto the ring

$$
\begin{aligned}
T=F\left\langle\xi, u_{1}, \ldots, u_{p-1}, v, y\right| \xi^{p}=0, \xi u_{1}-u_{1} \xi=1, \\
\left.\xi u_{i}-u_{i} \xi=u_{i-1}(i \geq 2), \xi v=v \xi, v y=y v=1\right\rangle
\end{aligned}
$$

whose inverse is induced by the map $u_{i} \mapsto d^{p-i-1}(x) y, v \mapsto d^{p-1}(x)$. Now we can write $T \cong\left(T_{1} *_{F} F[y]\right) /\langle v y-1, y v-1\rangle$, where $T_{1}=\left(T_{2} *_{F} F[v]\right) /\langle\xi v-v \xi\rangle$,

$$
T_{2}=\frac{F\left\langle u_{1}, \ldots, u_{p-1}\right\rangle[\xi, \delta]}{\left\langle\xi^{p}\right\rangle}
$$

and $\delta$ is the $k$-derivation given by $\delta\left(u_{i}\right)=u_{i-1}(i=2, \ldots, p-1)$ and $\delta\left(u_{1}\right)=1$. By Theorem 1 and Corollary 7, it follows that $T_{2} \cong \mathbf{M}_{p}(K)$, where $K=F\left\langle y_{1}, \ldots\right.$, $\left.y_{p^{2}-2 p+1}\right\rangle$. Applying Theorem 5 to $T_{1}$, we obtain $T_{1} \cong \mathbf{M}_{p}\left(K *_{F} F\left\langle z_{1}, \ldots, z_{p}\right\rangle\right)$. Since $v$ commutes with $\xi$, by Lemma 3 , the matrix $V$ corresponding to $v$ in $T_{1}$ is of the form $\sum_{i=1}^{p} z_{i} \Xi^{i-1}$, where $\Xi=\sum_{i=1}^{p-1} e_{i+1, i}$ is the matrix corresponding to $\xi$. So $V$ is a lower triangular matrix with $z_{1}$ on the entries of the main diagonal. The ring $T$ is obtained from $T_{1}$ by freely adjoining to it an inverse of $v$. But freely adjoining an inverse of $v$ to $T_{1}$ is equivalent to freely adjoining an inverse of $z_{1}$ to $K *_{F} F\left\langle z_{1}, \ldots, z_{p}\right\rangle$. Therefore, $T \cong \mathbf{M}_{p}\left(K *_{F} F\left\langle z_{2}, \ldots, z_{p}\right\rangle *_{F} F\left[z_{1}, z_{1}^{-1}\right]\right)$. And this proves the theorem.

\section{REFERENCES}

1. G. Agnarsson, S. A. Amitsur and J. C. Robson, Recognition of matrix rings II, Israel J. Math. 96 (1996), 1-13.

2. P. M. Cohn, Free rings and their relations, 2nd ed., London Mathematical Society Monographs No. 19 (Academic Press, 1985).

3. V. O. Ferreira, Tensor rings under field extensions, J. Algebra 231 (2000), 342-363.

4. L. Gerritzen, Taylor expansion of noncommutative polynomials, Arch. Math. (Basel) 71 (1998), 279-290.

5. T. de W. Jooste, Primitive derivations in free associative algebras, Math. Z. 164 (1978), 15-23. $381-410$.

6. V. K. Kharchenko, Constants of derivations of prime rings, Izv. Math. 18 (1982),

7. V. K. Kharchenko, Automorphisms and derivations of associative rings, Mathematics and Its Applications (Soviet Series) Vol. 69 (Kluwer Academic Publishers Group, 1991).

8. T. Y. Lam and A. Leroy, Recognition and computation of matrix rings, Israel $J$. Math. 96 (1996), 379-397. 